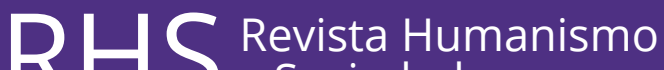 \\ y Sociedad
}

\section{La generación de identidad de marca mediante el uso de música en la publicidad de las cervezas Águila, Club Colombia y Pilsen}

\section{Brand identity generation by using music in advertising Águila, Club Colombia and Pilsen beers}

\author{
Andrés Alzate Ortiz ${ }^{1 *}$, Diana Zoraida Osorno López ${ }^{2 * *}$ \\ ${ }^{1}$ andresalzate82@hotmail.com; ${ }^{2}$ zora.osorno@gmail.com
}

El presente artículo surge del trabajo de grado realizado en el año 2015 para optar al grado de especialista en Gerencia de Mercadeo.

Recibido: noviembre 8 de 2015. Aceptado: diciembre 3 de 2015.

doi: 10.22209/rhs.v3n1.2a03

\section{Resumen}

Este artículo analiza si el sonido musical como herramienta del marketing sensorial usado por marcas de cervezas en Medellín, como Pilsen, Águila y Club Colombia, genera recordación e identidad de marca en personas entre 25 y 40 años. Compara las sensaciones emocionales generadas por la música usada en los anuncios de las marcas de cerveza, con las que la marca quiere transmitir. La metodología diseñada permite analizar música y letra usadas por las marcas; permite definir el mensaje real e identificar qué tipo de emociones transmiten las marcas, a partir de sus piezas musicales cantadas de carácter publicitario llamadas comercialmente jingles. Finalmente, se concluyó que el uso de la música en la publicidad de las marcas de cerveza genera identidad de marca. Las melodías simples y sencillas acompañadas de armonía tradicional generan fácil recordación. Esto le funciona adecuadamente a Pilsen, cuya música genera el mayor impacto y recordación.

Palabras clave: marketing sensorial, jingle, identidad de marca, cervezas, recordación.

\section{Abstract}

In this paper, we explore whether music as a sensorial marketing tool used by beer brands in Medellin, such as Pilsen, Águila and Club Colombia, produces memory recall and brand identity in people between 25 and 40 years old. We compare the feelings generated and transmitted by the music used in ads by these beer brands. The techniques we use allows us to analyze the music and lyrics employed and reveal the real message and emotions transmitted through musical jingles. Finally, we concluded that the use of music in advertising beer brands generates brand identity. Simple and easy melodies accompanied by traditional harmonies develops easy memory recall. This sensorial marketing works well with Pilsen, whose music produces the biggest impact and remembrance.

Keywords: sensorial marketing, jingle, brand identity, beers, remembrance.

Para citar este artículo: Alzate Ortiz, A. y Osorno López, D.Z. (2015). La generación de identidad de marca mediante el uso de música en la publicidad de las cervezas Águila, Club Colombia y Pilsen. Rev Humanismo y Sociedad, 3(1-2), 14-23. doi: 10.22209/rhs.v3n1.2a03

*Músico. Egresado de la Universidad EAFIT. Se ha desempeñado como docente de Música de la Universidad EAFIT, y director ejecutivo y productor musical de la empresa de producción de audio Ultrasonido Estudio S.A.S.

**Diseñadora industrial. Egresada de la Universidad Pontificia Bolivariana. 


\section{Introducción}

El marketing sensorial busca utilizar estímulos que afecten o generen sensaciones en los cinco sentidos: gusto, olfato, tacto, vista y oído. El personal encargado del mercadeo de las empresas entiende que el sonido y la música hacen parte de estos estímulos, y para desarrollar las piezas de audio o musicales, en especial, se contratan músicos y compositores bajo la guía de los publicistas o del mismo personal de mercadeo (Bernal y Santa Galvis, 2008). Las piezas de audio y musicales se componen bajo los parámetros objetivos de estas personas desde la intuición y experiencia profesional de cada uno.

El análisis y estudio de la música en la publicidad aún es muy incipiente. Palencia-Lefler (2009), profesor de música y publicidad de la Universidad Pompeu Fabra en Barcelona, destaca la importancia de analizar la música como «objeto de estudio», clasificando las diferentes formas musicales utilizadas en los medios publicitarios; esto lo hace con el fin de comprender las razones que los músicos, compositores, las agencias y los medios utilizan para tomar decisiones a la hora de construir una pieza musical publicitaria. Palencia recalca la importancia de la música en una publicidad audiovisual, dado que pensarlo sin música sería inconcebible, sin embargo, ni académicos ni publicistas parecen darle la importancia que merece y los estudios alrededor de ella y sus efectos en estas piezas son muy escasos, al igual que los recursos y presupuestos destinados para el audio en estos proyectos (Alpert \& Alpert, 1991).

En los puntos de venta, el sonido ocupa un espacio fundamental que influye en los consumidores; de esta manera, las reacciones de estos ante los estímulos musicales comienzan a ser utilizadas para experimentos en dichos lugares. Anthes (2010) analiza cómo algunas marcas utilizan música a un volumen demasiado alto, tan alto que ni se puede sostener una conversación. La estrategia de estas marcas es generar una sensación de fiesta constante, por ejemplo, pues son marcas con consumidores jóvenes receptivos a este tipo de estímulos.

Esta investigación busca analizar si el sonido como herramienta del marketing sensorial usado por las marcas de cervezas en la ciudad de Medellín, funciona como instrumento para generar recordación e identidad de marca en personas entre los 25 y 40 años de edad. Esto se logra, al analizar el impacto y nivel de recordación que genera el uso del sonido como elemento del marketing sensorial en los anuncios publicitarios de las marcas de cerveza en Medellín, específicamente, Pilsen, Águila y Club Colombia. También busca comparar las sensaciones encontradas, que genera la música usada en los anuncios de las marcas de cerveza.

\section{Metodología}

Partiendo de una metodología de tipo exploratorio y cualitativo, (Cook et al., 1986) se buscaba desarrollar un trabajo que permitiera identificar varios de los componentes en acción, que nos ayudaran a determinar los elementos relevantes, útiles, al momento de poder analizar la música y letra usadas por las marcas, y también permitir definir el mensaje real y las emociones que transmiten las marcas a partir de sus piezas musicales.

Se diseñaron herramientas para la recolección de la información necesaria dentro del proyecto que permitieran en cada una de las etapas de la metodología, lograr cumplir con los objetivos planteados dentro de la investigación.

En la figura 1 (en la siguiente página) podemos observar las etapas que se desarrollan en la metodología. Cada etapa responde a una necesidad de información que va a permitir concluir y responder la pregunta planteada. Además, se conecta con los objetivos del proyecto y a las actividades que se deben desarrollar para cumplir con los objetivos.

\section{Etapa de información}

Esta etapa permitió recolectar la información necesaria sobre las tres marcas de cervezas seleccionadas. Se buscaron los antecedentes, las características del producto, la estrategia publicitaria, el audio y mensaje que quiere transmitir la marca a través de todos sus esfuerzos comunicativos.

Partiendo de la construcción de un brief de marca desarrollado a partir del modelo propuesto por Rofes (2013), se logró entender con detalle cuáles son los mensajes de cada una de las marcas de cervezas seleccionadas. Se tomaron en cuenta los aspectos más importantes del producto como: antecedentes, ciclo de vida de la empresa, trayectoria histórica a nivel publicitario de la empresa, 


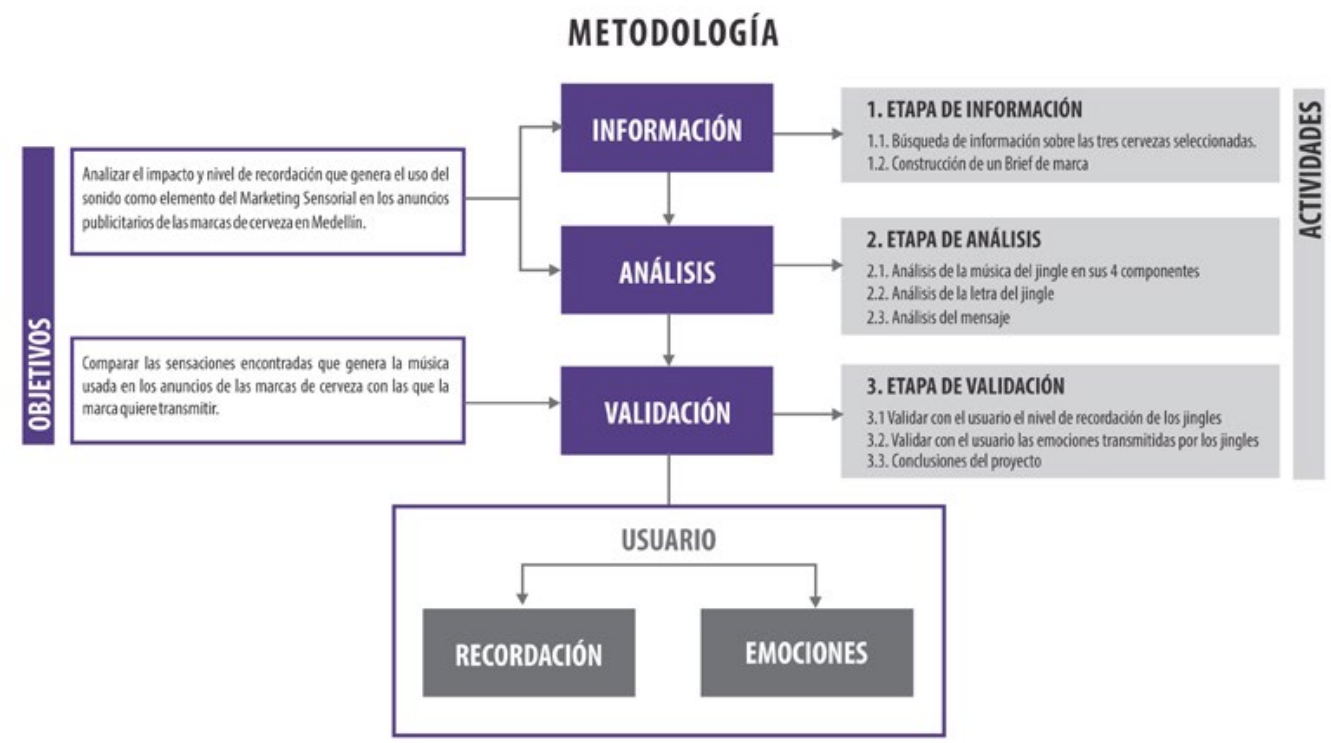

Figura 1. Metodología.

Fuente: elaboración propia.

personalidad de marca, audio y esfuerzos comunicativos, y fueron estos puntos los que permitieron una comparación clara con la competencia.

\section{Etapa de análisis}

Esta etapa se centró en el jingle, canción o melodía de corta duración y de fácil recordación (Pardavila Neira, 2009), seleccionado para cada marca de cerveza. Se realizó un análisis de tres elementos fundamentales del jingle, para cada elemento se diseñó una herramienta de análisis que permitiera entender y comparar los jingles de las diferentes cervezas.

La primera herramienta como muestra la figura 2, permitió estudiar la música del jingle en sus cuatro variables: melodía, ritmo, timbre y armonía (Balsebre, 1994). Para cada uno de sus componentes se analizaron elementos relevantes que pudieran arrojar datos de comparación.
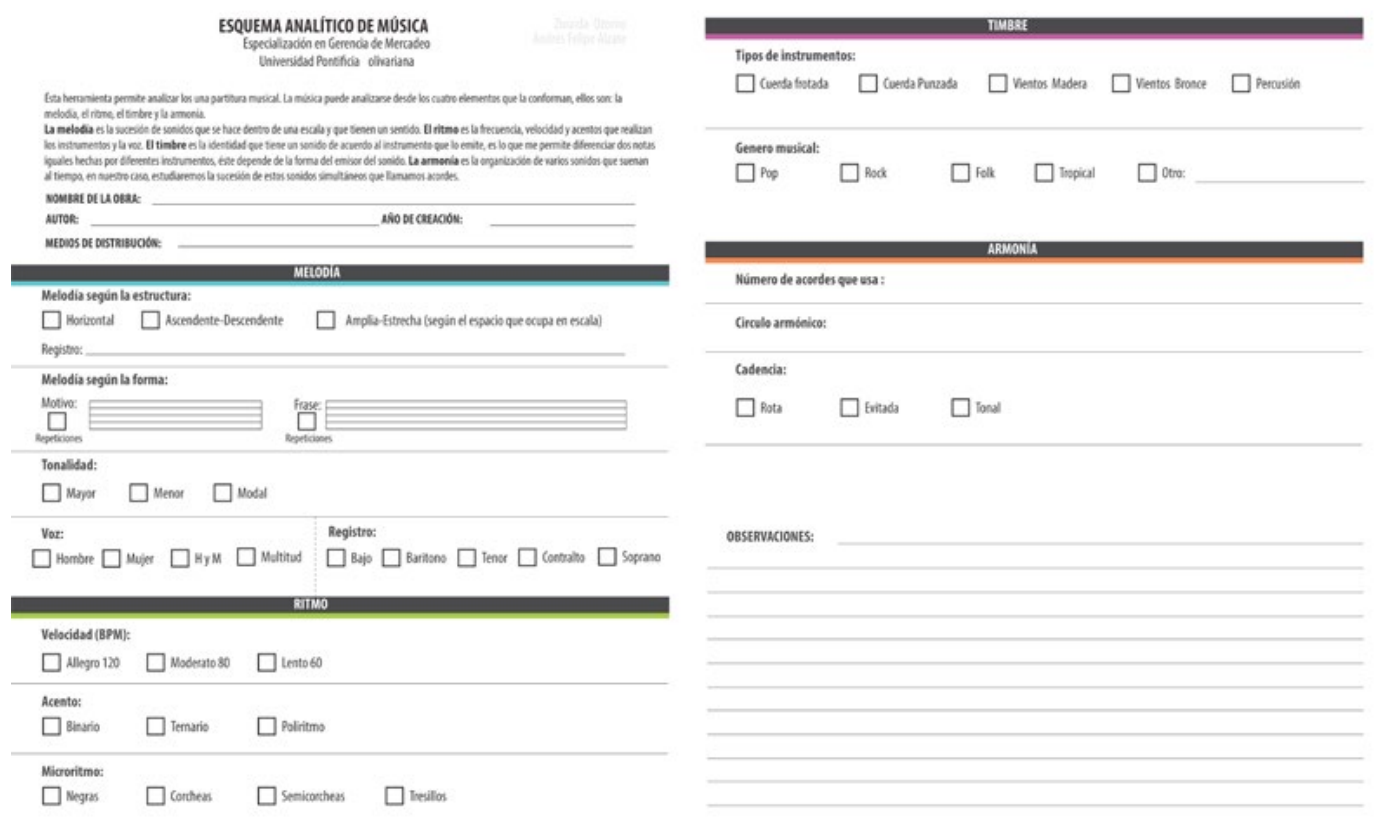

Figura 2. Metodología.

Fuente: elaboración propia. 
La segunda herramienta que se diseñó permitió analizar la letra de los jingles. El objetivo de esta herramienta era clasificar el texto por frases, buscar sentido, significados y palabras clave con las que estaba elaborado el

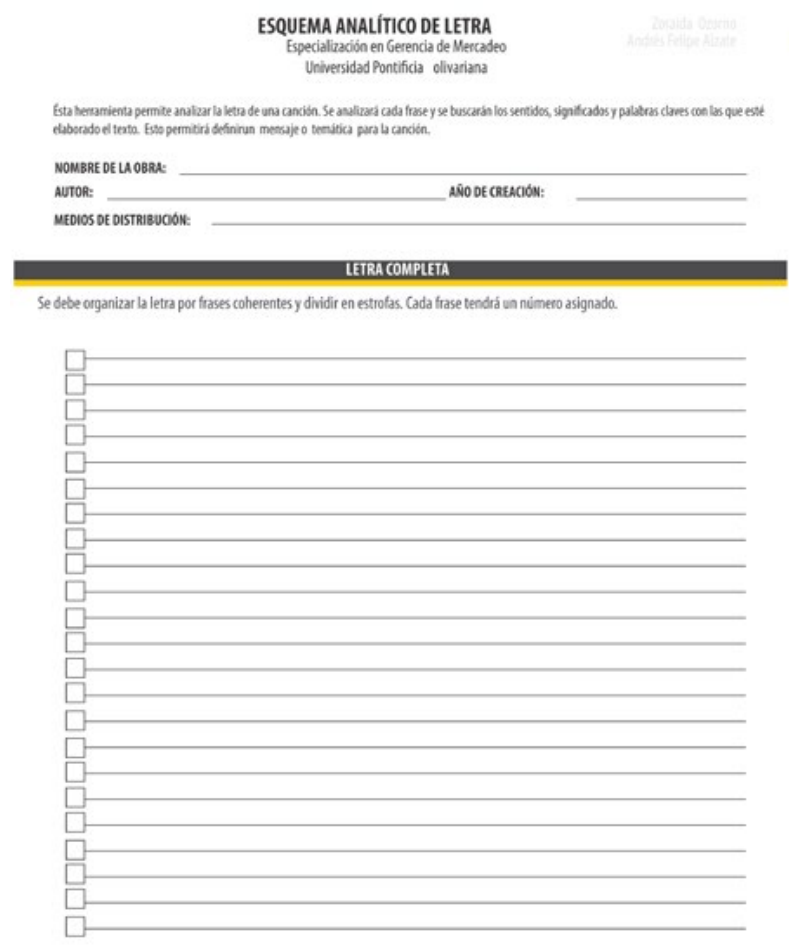

texto, que permitieran definir un mensaje o una temática para el jingle. La figura 3 muestra la herramienta y cómo para cada frase se debía encontrar los elementos claves y significativos.

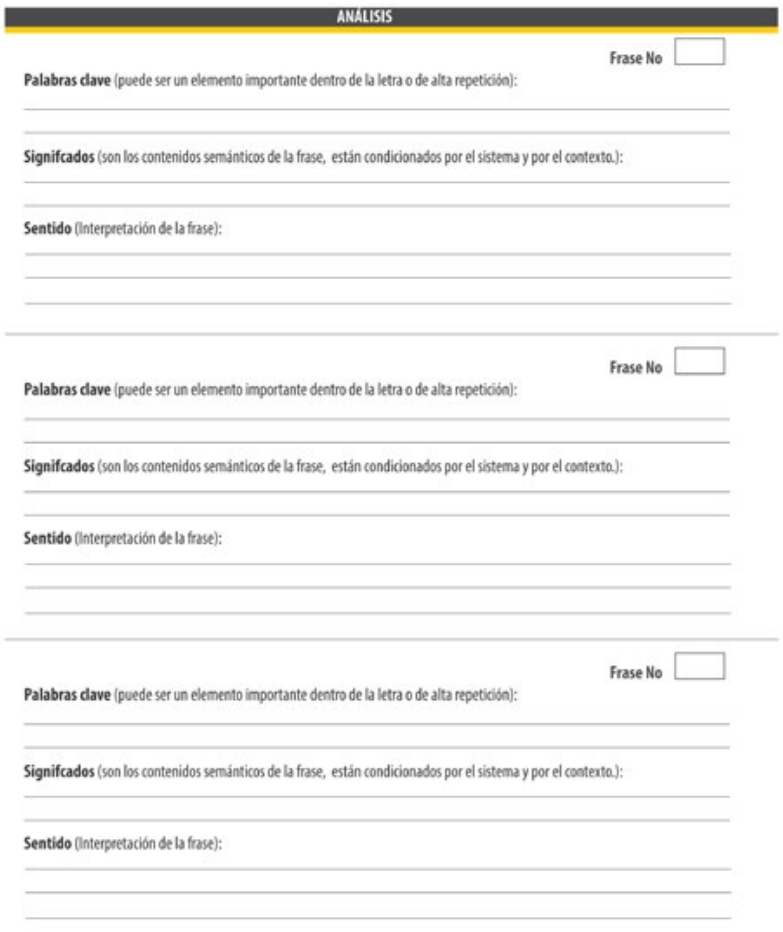

Figura 3. Herramienta de análisis de letra. Fuente: elaboración propia.

\section{Etapa de validación}

En esta etapa se confrontó al usuario con cada uno de los jingles; se manejó en dos niveles. El primer nivel comprobó el grado de recordación que tiene el jingle. El segundo nivel asoció emociones determinadas a cada uno de ellos, (Arregui Borrero, 2015). Esta prueba se realizó en un estudio de grabación, donde el usuario pudo escuchar con claridad, tranquilidad y aislado del mundo exterior cada uno de los jingles, mientras el usuario escuchaba la canción respondía un check list de recordación diseñado para la validación del proyecto. La figura 4 (en la siguiente página) muestra la herramienta utilizada en la etapa de validación.

\section{Resultados}

Se analizaron las tres marcas, y se construyó un brief bastante completo de cada marca, donde se miraron aspectos como antecedentes, ciclo de vida de la empresa, nivel publicitario, audio y esfuerzos comunicativos entre otros ítems, que fueron pieza clave para construir la herramienta y lograr el propósito de tener un análisis detallado y lograr comparativos relevantes. El análisis de las tres marcas elegidas para esta investigación (etapa de información) nos mostró que Pilsen siempre busca comunicar el sentido de la amistad como un pilar estratégico, de una manera fresca con un lenguaje sencillo y muy cercano.

El consumidor es clave, la materia prima que usa Pilsen para construir su propuesta va más allá de un rango de edad, de un nivel socioeconómico determinado, ya que lo importante es el valor otorgado a reunirse con los amigos para compartir una cerveza. Pilsen es la marca de los «parceros», del «parche»; es la cerveza que en su momento estuvo a «mil-cua»y «mil-qui», la 


\section{CHECK LIST DE RECORDACIÓN \\ Especialización en Gerencia de Mercadeo}

Universidad Pontificia olivariana

La siguiente lista de chequeo permite evaluar que tanta recordación genera una marca a partir de su jingle o tema musical promocional.

NOMBRE COMPLETO:

EDAD: OCUPACIÓN: TELÉFONO:

A continuación usted escuchará diferentes temas musicales. Cada tema está enumerado y tiene un espacio para que usted responda si recuerda o no la marca o el producto con el que se promociona ese tema musical y otro espacio para que dé el nombre del producto solo si lo recuerda.

TEMA MUSICAL No1
1.1. ¿Há escuchado al guna vez esta música?
1.2. ¿Conoce usted el producto al que hace referencia éste tema musical?
1.3. Escriba el nombre del producto si lo recuerda:

\section{TEMAMUSICAL No2}

TEMA MUSICAL No2
2.1. ¿Há escuchado alguna vez esta música?
2.2. ¿Conoce usted el producto al que hace referencia éste tema musical?
2.3. Escriba el nombre del producto si lo recuerda:

\section{TEMA MUSICAL No 3}
3.1. ¿Há escuchado alguna vez esta música?
SI
NO
3.2. ¿Conoce usted el producto al que hace referencia éste tema musical?
SI $\square$ No
3.3. Escriba el nombre del producto si lo recuerda:

Figura 4. Check list de validación. Fuente: elaboración propia.

cerveza de los héroes, la cerveza apropiada para consumir luego de una jornada laboral.

Por otro lado, Cerveza Águila es una marca que refleja alegría, rumba, compartir con los amigos, diversión y motivos de celebración: donde hay Águila, hay rumba. Todo el tiempo Águila se hace presente en las fiestas y carnavales del país, mostrando por qué Águila es Colombia, usando el tricolor en la etiqueta, aplicativos de diseños de personajes como el de Joe Arroyo en una edición limitada en presentación enlatada. Emplea diseños que adecúan la marca a cada evento, como la temporada del mundial de Fútbol, los carnavales de Barranquilla, Festival Vallenato, Carnaval de Negros y Blancos.

Cerveza Club Colombia es una marca que comunica estatus, buen gusto, estilo, porte, elegancia, encanto, seducción. En uno de sus eslóganes más recordados, es catalogada como «perfecta». La Cerveza Club 
Colombia, al comunicar estilo y buen gusto, se hace presente como acompañamiento de una buena cena, en reuniones de negocios y de amigos, destacando siempre su categoría y creando un ambiente de elegancia.

Durante la etapa de análisis, se encontraron similitudes en la melodía de las piezas de Pilsen y Cerveza Águila, ambas utilizan un registro medio y melodías sencillas pertenecientes a la tonalidad. La tonalidad de ambas piezas es mayor, para la pieza de Pilsen se tiene: re mayor, y para la pieza de Cerveza Águila: la mayor.

Se presentaron diferencias entre la instrumentación (timbre) de las tres piezas de audio. Pilsen usa percusión electrónica (en su mayoría sonidos secuenciados), por el contrario, Cerveza Águila se apoya en sonidos emitidos por instrumentos característicos de la música folclórica colombiana. Club Colombia utiliza instrumentos de viento como la trompeta con sordina, y la guitarra acústica en un estilo Jazz.

La letra representa un elemento importante de esta mezcla de sonidos. Encontramos coherencia en
Cerveza Águila: «Águila es Colombia, refresca nuestra pasión». Esta letra va con la personalidad de la marca analizada en la etapa de información. La letra de Club Colombia también mostró coherencia con la metodología de información: «Las mejores cosas de la vida toman tiempo», además de usar un estilo de música más complejo que el de Pilsen y Cerveza Águila, como el Jazz que comunica estilo y buen gusto. Por su parte, Pilsen con el texto: «Siempre Pilsen va... marcando el paso», no muestra coherencia con la información de la marca y lo que esta quiere transmitir.

En la etapa de validación, se expuso a una muestra de 60 personas entre los 25 y 40 años de edad de la ciudad de Medellín, las tres piezas de audio y una encuesta que arrojó los siguientes resultados.

E1 98.3\% dice haber escuchado alguna vez la música de Pilsen y el $95 \%$ dice conocer el producto al que hacía referencia ese audio, sin embargo, solo el $73 \%$ reconoció que efectivamente la música era de Pilsen; Cerveza Águila fue el producto con el que más fue confundida (Figura 5 y Figura 6).

¿Ha escuchado alguna vez esta música?

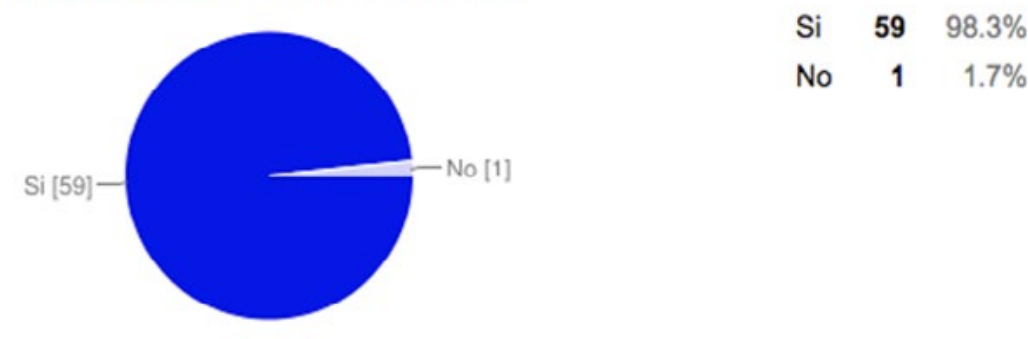

Figura 5. Encuesta con el jingle de Pilsen. Fuente: elaboración propia.

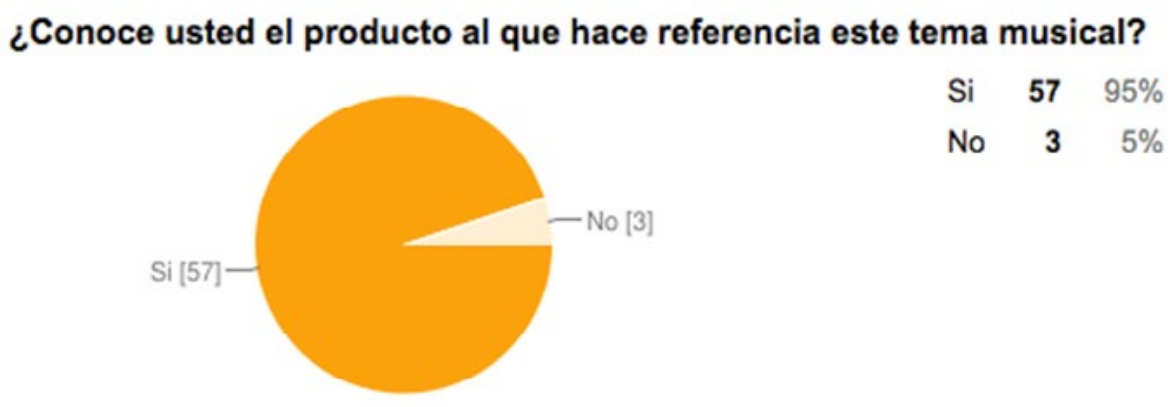

Figura 6. Encuesta con referencia al tema musical Pilsen. Fuente: elaboración propia. 
La emoción más representativa con respecto a la música de Pilsen fue alegría con un $53.3 \%$ y entusiasmo con
$26.7 \%$. Este resultado fue coherente con la identidad de la marca (Figura. 7).

\section{Con cuál de estas emociones asocia esta pieza musical}

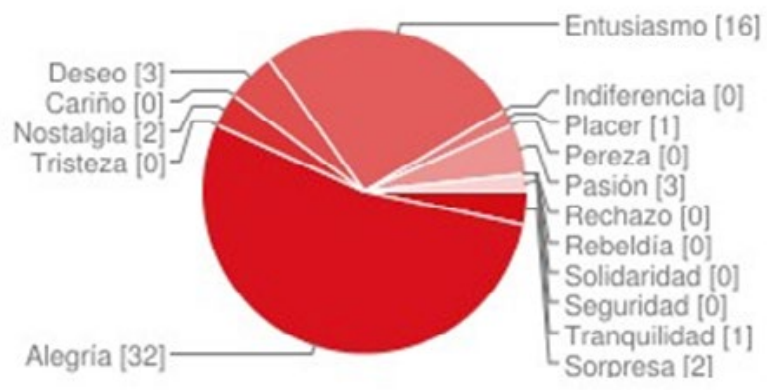

$\begin{array}{lrr}\text { Sorpresa } & \mathbf{2} & 3.3 \% \\ \text { Alegria } & \mathbf{3 2} & 53.3 \% \\ \text { Tristeza } & \mathbf{0} & 0 \% \\ \text { Nostalgia } & \mathbf{2} & 3.3 \% \\ \text { Cariño } & \mathbf{0} & 0 \% \\ \text { Deseo } & \mathbf{3} & 5 \% \\ \text { Entusiasmo } & \mathbf{1 6} & 26.7 \% \\ \text { Indiferencia } & \mathbf{0} & 0 \% \\ \text { Placer } & \mathbf{1} & 1.7 \% \\ \text { Pereza } & \mathbf{0} & 0 \% \\ \text { Pasión } & \mathbf{3} & 5 \% \\ \text { Rechazo } & \mathbf{0} & 0 \% \\ \text { Rebeldia } & \mathbf{0} & 0 \% \\ \text { Solidaridad } & \mathbf{0} & 0 \% \\ \text { Seguridad } & \mathbf{0} & 0 \% \\ \text { Tranquilidad } & \mathbf{1} & 1.7 \%\end{array}$

Figura 7. Piezas musicales.

Fuente: elaboración propia.

La música de Pilsen fue relacionada con rumba, amigos, fútbol, mujeres, playa, fiesta, diversión, infancia, héroe, viernes de relajo, baile, entre otras sensaciones. Podemos analizar aquí la razón por la cual algunos confunden este producto con Cerveza Águila, ya que la identidad de Águila es playa, fútbol, mujeres. Esta música, al estar relacionada con estos momentos, condujo a que algunos de los encuestados hicieran referencia a Águila, aunque esta música pertenecía en realidad a Pilsen.
El $86.7 \%$ de los encuestados dice haber escuchado alguna vez la música de Águila, el 78\% dice conocer el producto al que hace referencia esta música; pero solo el $56 \%$ escribió el producto correcto (Figura 8 y Figura 9). Las marcas con las cuales se confundió el producto fueron Pilsen, Cola y Pola, Aguardiente Néctar, entre otros.

\section{¿Ha escuchado alguna vez esta música?}

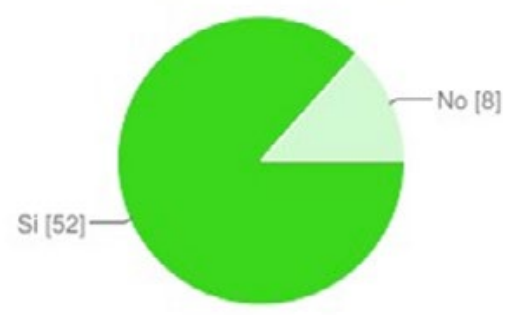




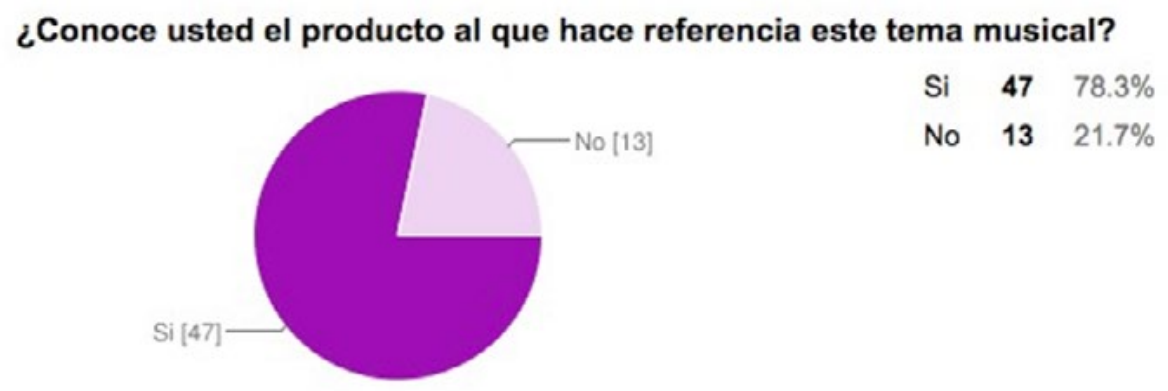

Figura 9. Encuesta 2 con el Jingle de Águila.

Fuente: elaboración propia.

La emoción más representativa asociada con la música de Cerveza Águila fue Alegría con un 33.9\% seguida por pasión $25.4 \%$ y entusiasmo con $26.7 \%$
(Figura 10). Este resultado fue coherente con la identidad de marca.

\section{Con cuál de estas emociones asocia esta pieza musical}

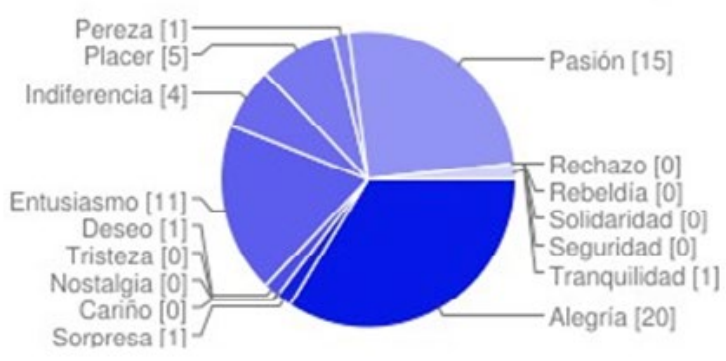

$\begin{array}{lrr}\text { Alegria } & \mathbf{2 0} & 33.9 \% \\ \text { Sorpresa } & \mathbf{1} & 1.7 \% \\ \text { Tristeza } & \mathbf{0} & 0 \% \\ \text { Nostalgia } & \mathbf{0} & 0 \% \\ \text { Cariño } & \mathbf{0} & 0 \% \\ \text { Deseo } & \mathbf{1} & 1.7 \% \\ \text { Entusiasmo } & \mathbf{1 1} & 18.6 \% \\ \text { Indiferencia } & \mathbf{4} & 6.8 \% \\ \text { Placer } & \mathbf{5} & 8.5 \% \\ \text { Pereza } & \mathbf{1} & 1.7 \% \\ \text { Pasión } & \mathbf{1 5} & 25.4 \% \\ \text { Rechazo } & \mathbf{0} & 0 \% \\ \text { Rebeldia } & \mathbf{0} & 0 \% \\ \text { Solidaridad } & \mathbf{0} & 0 \% \\ \text { Seguridad } & \mathbf{0} & 0 \% \\ \text { Tranquilidad } & \mathbf{1} & 1.7 \%\end{array}$

Figura 10. Encuesta 3 con el Jingle de Águila. Fuente: elaboración propia.

La música de Águila fue relacionada con la selección Colombia, fútbol, estadio, vuvuzela, playa, día soleado, pasión, fiesta con amigos unidos por una causa, nacionalismo, Cartagena. Estas situaciones son coherentes con la identidad de la Cerveza Águila.
El $90 \%$ de las personas encuestadas dice haber escuchado alguna vez la música de Club Colombia, y el $83.3 \%$ dice conocer el producto al que hace referencia este tema musical (Figura 11 y Figura 12). Sin embargo, solo el $60 \%$ eligió a Club Colombia como el producto al que hacía referencia el tema musical. 


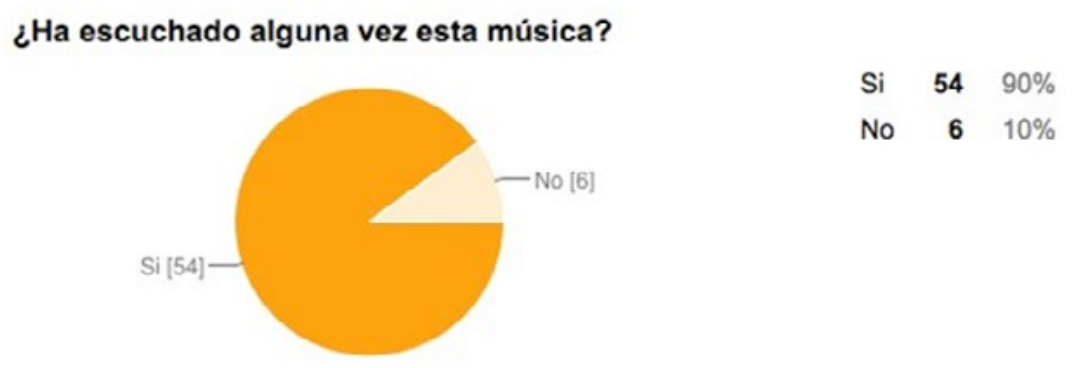

Figura 11. Encuesta 1 con el Jingle de Club Colombia. Fuente: elaboración propia.

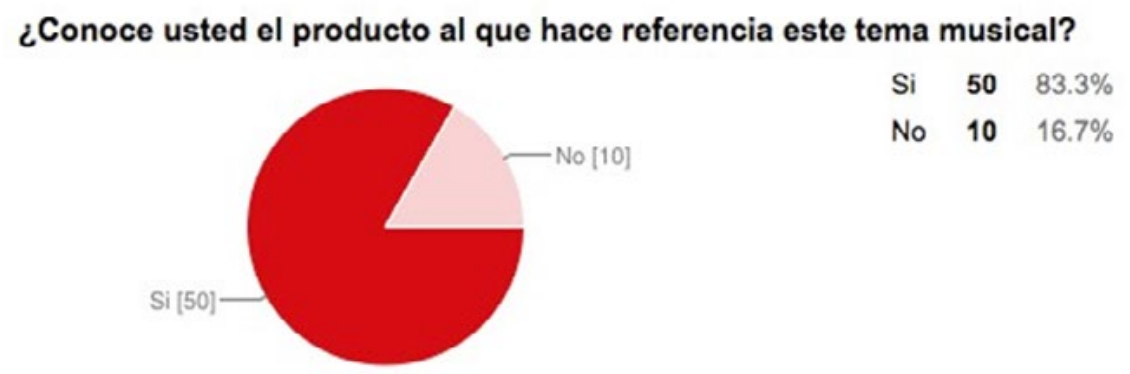

Figura 12. Encuesta 2 con el Jingle de Club Colombia.

Fuente: elaboración propia.

La emoción más representativa asociada con la música de Club Colombia fue placer con $46.6 \%$, seguida de tranquilidad con $15.5 \%$ (Figura 13). Estas emociones son coherentes con la identidad de la marca.

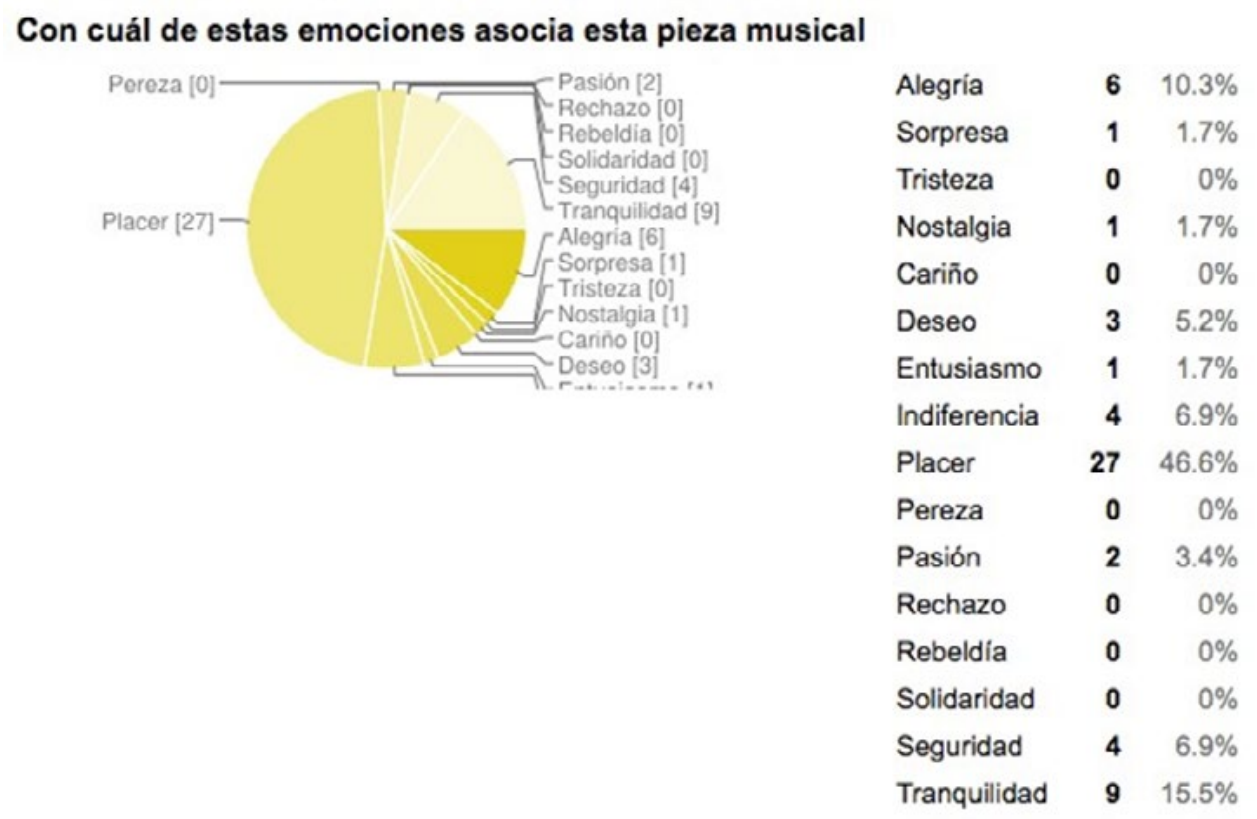

Figura 13. Encuesta 3 con el Jingle de Club Colombia. Fuente: elaboración propia. 
Las emociones y las sensaciones transmitidas por esta música acertaron con la identidad de la marca: cena elegante, comer algo delicioso, sitio agradable, buena música, producto elaborado, burbujas, relajo, exclusividad, clase, elegancia, cerveza premium, seducción, entre otras.

\section{Conclusiones}

El uso de la música en la publicidad de las marcas de cerveza genera identidad de marca. En este estudio, sin pronunciar el nombre de la marca y sin la ayuda de ninguna imagen visual, la mayoría de los encuestados eligieron la marca a la que hacía referencia el producto y las sensaciones y emociones que les producía van de la mano con lo que la marca quiere comunicar.

La música con mayor impacto y recordación fue Pilsen, sin embargo, el texto usado en la pieza no es coherente con lo que quiere comunicar. Podemos decir que la melodía sencilla y la armonía simple (dos acordes) generan más recordación que sus dos competidoras en el estudio. Esto, pues, se podría generalizar diciendo que las melodías simples y sencillas acompañadas de armonía tradicional generan fácil recordación.

La música de estas piezas analizadas produce unas sensaciones similares en la mayoría de los encuestados, por lo que podemos concluir que según el significado que tienen estos géneros musicales para los consumidores de cerveza de la ciudad de Medellín, el objetivo de comunicación de las piezas se cumple.

Finalmente, es necesario resaltar que el género musical es importante a la hora de comunicar una marca, no es lo mismo el Jazz usado por Club Colombia, que la música folclórica de Cerveza Águila.

\section{Referencias}

Alpert, J.I., y Alpert, M.I. (1991). Contributions from a musical perspective on advertising and consumer behavior. Advances in Consumer Research, 18(1), 232-238.

Anthes, E. (2010). It's so loud, I can't hear my budget. Psychology Today, 43 (5), 40.

Arregui Borrero, A. B. (2015). El jingle y las canciones como estrategia de posicionamiento de marca. Casos de estudio: Parmalat, Banco del Pacífico, Coca Cola (Tesis de pregrado en comunicación). Universidad Internacional SEK, Ecuador. Recuperado de http://repositorio.uisek.edu.ec/jspui/handle/123456789/1147

Balsebre, A. (1994). El lenguaje radifónico. Madrid: Cátedra, 93.

Bernal, A. E., y Santa Galvis, J. (2008). El marketing sensorial como herramienta para el fortalecimiento de la imagen corporativa (Tesis de pregrado de Comunicación Social). Pontificia Universidad Javeriana, Bogotá. Recuperado de http://javeriana.edu.co/biblos/tesis/comunicacion/tesis106.pdf

Cook, T.D. \& Reichardt, C. S. (1986). Métodos cualitativos y cuantitativos en investigación evaluativa. (Guillermo Solana, trad.). Madrid: Ediciones Morata. (Obra original publicada en 1982)

Palencia-Lefler, M. (2009). La música en la comunicación publicitaria (Music in the advertising communication). Comunicación y sociedad, 22 (2), 89-108.

Pardavila Neira, M. (2009) Las primeras creaciones musicales para publicidad en Radio y Televisión en España: El Jingle (1959-1960). En Olarte Martínez, M. (Ed.). (2009) Reflexiones en torno a la música y la imagen desde la Musicología española. Salamanca: Plaza Universitaria Ediciones.

Rofes, T. S. (2013). El brief de la Marca España. Visual: magazine de diseño, creatividad gráfica y comunicación, (164), 41-49. 